\title{
Numerical studies of confinement in the Landau gauge by the larger lattice simulation
}

\author{
Hideo Nakajima* \\ Department of Information Science, Utsunomiya University, 321-8585 Japan \\ Sadataka Furui ${ }^{\dagger}$ \\ School of Science and Engineering, Teikyo University, 320-8551 Japan
}

\begin{abstract}
The infrared behaviour of QCD in the Landau gauge is studied in $24^{4}$ and $32^{4}$ lattices by measuring the Kugo-Ojima parameter, gluon propagator, ghost propagator and the QCD running coupling. The Kugo-Ojima parameter c remains about 0.8 instead of the expectation value 1 . We observe infrared suppression of the singularity of the gluon propagator and enhancement of the ghost propagator but the shifts are smaller than those of Dyson-Schwinger approach. The momentum dependence of the running coupling in the infrared region is weak and $\alpha_{s}(0) \simeq 1$.
\end{abstract}

\section{The infrared behaviour of QCD in the Landau gauge}

The colour confinement in QCD can be formulated as an absence of coloured asymptotic states in the BRST formalism, and the presence of longrange $1 / p^{4}$ correlation between colour sources. In the Gribov-Zwanziger's theory of Landau gauge $\mathrm{QCD}[1]$, the long-range correlation is attributed to the ghost propagator, i.e. the inverse of the Faddeev-Popov(FP) matrix. The ghost propagator is more singular and the gluon propagator is less singular than the tree level in the infrared region.

In Kugo-Ojima's theory[2], a sufficient condition for an absence of coloured asymptotic states is that the parameter $u^{a b}$ defined by the two-point function of the FP ghost fields, $c(x), \bar{c}(y)$, and $A_{\nu}(y)[3]$, satisfies $u^{a b}(0)=-\delta^{a b}$.

\section{The Kugo-Ojima confinement criterion and the Gribov-Zwanziger's theory}

Let two-point tensor $G_{\mu \nu x y}{ }^{a b}$ be

$G_{\mu \nu x y}^{a b}=\operatorname{tr}\left(\lambda^{a \dagger} D_{\mu} \frac{1}{-\partial D}\left(-D_{\nu}\right) \lambda^{b}\right)_{x y}$.

\footnotetext{
*e-mail nakajima@is.utsunomiya-u.ac.jp

${ }^{\dagger}$ e-mail furui@liberal.umb.teikyo-u.ac.jp
}

and the horizon function $H(U)$

$H(U)=\sum_{x, y, a} G_{\mu \mu x y}^{a a}-\left(N^{2}-1\right) E(U)$

where $N$ is the number of colours, now $N=3$. $E(U)$ depends on the options of the definition of gauge field, $U$-linear or $\log U$ type;

$U$-linear: $E(U)=\sum_{l} \frac{1}{N} \operatorname{Re} \operatorname{tr} U_{l}$,

$\log U: E(U)=\sum_{l, a} \operatorname{tr}\left(\lambda^{a \dagger} S\left(\mathcal{A}_{l}\right) \lambda^{a}\right) /\left(N^{2}-1\right)$, where $\mathcal{A}_{l}=a d j_{A_{l}}$, and $S(x)=\frac{x / 2}{\operatorname{th}(x / 2)}$.

One can show that in the core region[1], the horizon function $H(U)$ is negative and in the infinite volume limit $\lim _{V \rightarrow \infty}\langle H(U)\rangle / V=0$ which is called horizon condition.

Zwanziger showed in his lattice formulation that the ghost self-energy cancels precisely the zero-th order $p^{2}$ term and the ghost propagator behaves as $1 / p^{4}$ in the infrared asymptotic region provided no additional non-perturbative effect is involved [1]. Recent results of Dyson-Schwinger approach suggest the nonperturbative aspects are in effect.

Kugo also showed in the continuum formulation that the ghost propagator $G\left(p^{2}\right) / p^{2}$ behaves in the infrared asymptotic region as[2]

$G\left(p^{2}\right) / p^{2} \sim 1 /\left[p^{2}\left(1+u(0)+O\left(p^{2}\right)\right)\right]$

where $u\left(p^{2}\right)^{a b}=\delta^{a b} u\left(p^{2}\right)$. Thus Kugo-Ojima condition leads to the same singular behavior as 
Table 1

The Kugo-Ojima parameter $c$, trace $e / d$ and the horizon function deviation factor $h$ in $U$-linear(suffix $1)$ and $\log U($ suffix 2$)$ version, $\beta=6.0$. The bottom line is of $\beta=6.4$.

\begin{tabular}{l|lll|lll}
\hline$L$ & $c_{1}$ & $e_{1} / d$ & $h_{1}$ & $c_{2}$ & $e_{2} / d$ & $h_{2}$ \\
\hline 16 & $0.576(79)$ & $0.860(1)$ & -0.28 & $0.628(94)$ & $0.943(1)$ & -0.32 \\
24 & $0.695(63)$ & $0.861(1)$ & -0.17 & $0.774(76)$ & $0.944(1)$ & -0.17 \\
32 & $0.706(39)$ & $0.862(1)$ & -0.15 & $0.777(46)$ & $0.944(1)$ & -0.16 \\
\hline 32 & $0.650(39)$ & $0.883(1)$ & -0.23 & $0.700(42)$ & $0.953(1)$ & -0.25 \\
\hline
\end{tabular}

Zwanziger's, and derives the universal renormalizaiton factors as $\frac{Z_{1}}{Z_{3}}=\frac{1}{\tilde{Z}_{3}}=1+u(0)=0$.

Putting Kugo-Ojima parameter as $u(0)=-c$, one finds that the horizon condition is written as $\left(\frac{e}{d}\right)+(d-1) c-e=(d-1)\left(c-\frac{e}{d}\right)=(d-1) h=0$. where $e=E / V$ and $d=4$.

Thus one sees that both the above arguments appear to be consistent with each other provided the lattice covariant derivative meets with the continuum one in the naive sense, i.e., $e / d=1$.

Kugo discussed in his lecture[2] that the colour confinement can be realized via either (1) $Z_{1}=0$ and $Z_{3}=$ finite or (2) $Z_{1} / Z_{3}=0$ but/and $Z_{3}=0$

Zwanziger $[1,4]$ argues that the second possibility is realized in the $\mathrm{SU}(2)$ colour case.

Our numerical results are summarized in Table 1 . We obtained that $u_{b}^{a}(0)$ is consistent to $-c \delta_{b}^{a}, c=0.8$ in $S U(3)$ quenched simulation, $\beta=6.0$, on $24^{4}$ and $32^{4}$.

In the case of $\beta=6.4$ on $32^{4}$, the value of $e / d$ becomes closer to 1 , but $|h|$ becomes larger as compared to those of $\beta=6.0$, and thus $c$ becomes smaller. We need to perform larger lattice simulation to approach the continuum limit.

\section{The two-point functions (gluon propa- gator, ghost propagator)}

We measured gluon propagator of $\beta=6.0$ on lattice $16^{4}, 24^{4}$ and $32^{4}$, and observed clear tendency of the infrared suppression in the case of $24^{4}$ and $32^{4}$.

The ghost propagator is infrared divergent and its singularity can be parametrised as $p^{-2\left(1+\alpha_{G}\right)}$, where $p^{2}=\sum_{\mu}\left(4 \sin ^{2} \frac{\pi k_{\mu}}{L}\right),\left(-L / 2<k_{\mu} \leq L / 2\right)$.

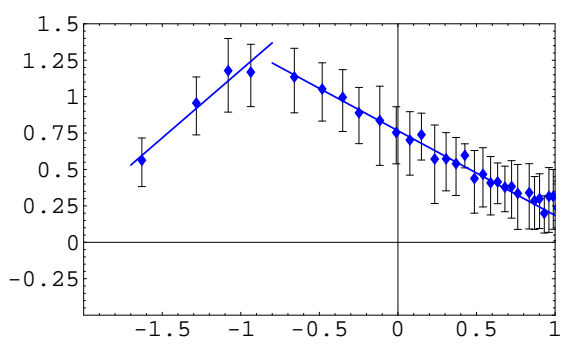

Figure 1. The log of the gluon dressing function as a function of the log of the lattice momentum pa. $\beta=6.0,32^{4}$ in $U$-linear version.

In the $32^{4}$ lattice, the overall scale difference in the $\log U$ and $U$-linear versions disappear in gluon propagator, but it still remains in ghost propagator.

The gluon propagator and the ghost propagator are evaluated also from Dyson-Schwinger approach[5]. They found qualitatively similar solutions for dressing functions of the gluon and the ghost, $Z\left(p^{2}\right) \sim\left(p^{2}\right)^{-\alpha_{D}}$ and $G\left(p^{2}\right) \sim\left(p^{2}\right)^{-\alpha_{G}}$ with $\alpha_{D}+2 \alpha_{G}=0, \alpha_{G} \simeq 0.595$ and $\alpha_{D} \simeq$ $-1.19[5]$.

The gluon propagator vanishes at 0 momentum if $\alpha_{G}>0.5$. Our lattice simulation of the ghost dressing function $G\left(p^{2}\right)$ shows that the value $\alpha_{G} \sim 0.18$ remains unchanged at $1 / 3$ of the Dyson-Schwinger one through $16^{4}$ to $32^{4}$ simulations. The negative $\alpha_{D}$ appears in $24^{4}$ lattice and $\alpha_{D} \simeq-0.4$ in $32^{4}$ lattice. We observe the tendency that $\left|\alpha_{D}\right|$ increases as the lattice size 

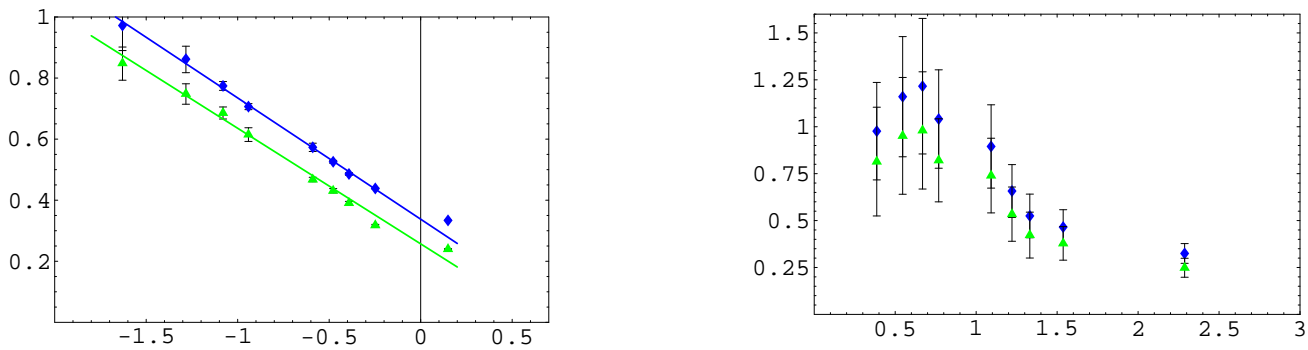

Figure 2. The log of the ghost dressing function as a function of the log of the lattice momentum pa(left) and the running coupling $\alpha_{s}$ as a function of the lattice momentum $p(\mathrm{GeV})$ (right). Triangles are in $\log U$ and diamonds are in $U$-linear version of $\beta=6.0,32^{4}$ lattice.

increases, but our data still stays about $1 / 3$ of the prediction of the Dyson-Schwinger approach.

In contrast to the first data of the ghost propagator[6], suppression at the lowest momentum is not seen in our data of the ghost propagator. For calculation of the ghost propagator, we adopted the multigrid Poisson solver whose accuracy was kept within $10^{-5}$, and we set $1 \%$ as an ending condition of perturbative calculation of the inverse FP operator. The Landau gauge accuracy of $\operatorname{div} A=0$ is $10^{-4}$ in maximum norm.

Using the ghost and gluon dressing function, the running coupling can be evaluated from $\alpha_{s}(p a)=\left(g^{2}(a) / 4 \pi\right) Z(p a) G(p a)^{2} \sim$ $(p a)^{-2 \alpha_{D}-4 \alpha_{G}}$, where $a$ is the lattice spacing. In the case of $\beta=6,32^{4}$, which has the smallest $|h|$, the suppression of the gluon dressing function due to the loss of correlation of the gluon field and the enhancement of the ghost dressing function cancel. We find $\alpha_{D}+2 \alpha_{G} \simeq 0$ near the lowest momentum point and $\alpha_{s}(0) \simeq 1$. The infrared suppression of the running coupling measured from the three point function of gluon[7] and from the ghost-antighost gluon[8] in $\overline{M O M}$ scheme would be attributed to the loss of correlation of gluon fields.

\section{Discussion and outlook}

In the $\beta=6,32^{4}$ lattice simulation $|h|$ is about 0.15 and we need extrapolation to $|h|=0$. There remain finite size effects in ghost propagator in $32^{4}$ lattice, and also in the Kugo-Ojima parameter which manifest themselves in their dependence on the definition of the gauge field. The continuum limit should not depend on the definition of the gauge field and so a systematic method for extrapolating the continuum limit of $\mathrm{c}$ in the two definitions is necessary.

This work was supported by the KEK supercomputing project No.02-82.

\section{REFERENCES}

1. D. Zwanziger, Nucl. Phys. B364 (1991) 127, Nucl. Phys. B412 (1994) 657.

2. T. Kugo, Int. Symp. on BRS symmetry, Kyoto, Sept. 18-22, 1995, hep-th/9511033.

3. H. Nakajima, S. Furui, A. Yamaguchi, Nucl. Phys. B (Proc. Suppl.) 94 (2001) 558, heplat/0010083 v2.

4. A. Cucchieri and D. Zwanziger, Phys. Lett. B524 123 (2002), hep-lat/0012024 v2.

5. C. S. Fischer, R. Alkofer and H. Reinhardt, hep-ph/0202195.

6. H. Suman and K. Schilling, Phys. Lett. B373 (1996) 314. 
7. Ph. Boucaud et al., hep-ph/9810322, 9810437

8. S. Furui and H. Nakajima, Confinement IV proc, hep-lat/0012017. 